\title{
Zileuton suppresses cholangiocarcinoma cell proliferation and migration through inhibition of the Akt signaling pathway
}

This article was published in the following Dove Press journal:

OncoTargets and Therapy

\author{
Sasikamon Khophai ${ }^{1,2}$ \\ Malinee Thanee ${ }^{1,2}$ \\ Anchalee Techasen ${ }^{2,3}$ \\ Nisana Namwat ${ }^{1,2}$ \\ Poramate Klanrit ${ }^{1,2}$ \\ Attapol Titapun ${ }^{2,4}$ \\ Apiwat Jarearnrat ${ }^{2,4}$ \\ Prakasit Sa-Ngiamwibool ${ }^{2,5}$ \\ Watcharin Loilome ${ }^{1,2}$ \\ 'Department of Biochemistry, \\ Faculty of Medicine, Khon Kaen \\ University, Khon Kaen, Thailand; \\ ${ }^{2}$ Cholangiocarcinoma Research \\ Institute, Khon Kaen University, \\ Khon Kaen, Thailand; ${ }^{3}$ Faculty of \\ Associated Medical Sciences, Khon \\ Kaen University, Khon Kaen, Thailand; \\ ${ }^{4}$ Department of Surgery, Faculty of \\ Medicine, Khon Kaen University, \\ Khon Kaen, Thailand; ${ }^{5}$ Department of \\ Pathology, Faculty of Medicine, Khon \\ Kaen University, Khon Kaen, Thailand
}

Correspondence: Watcharin Loilome Department of Biochemistry, Faculty of Medicine, Khon Kaen University, 123 Mittraparp Road, Muang District, Khon Kaen 40002, Thailand

Tel +6643363265

Fax +66 43348386

Email watloi@yahoo.com
Background: Inflammatory lipid mediators play an important role in several cancer types. Leukotrienes (LTs), pro-inflammatory lipid mediators, are involved in chronic inflammation and cancer progression. They are derived from arachidonic acid by 5-lipoxygenase (5-LOX) activity. On the other hand, 15-lipoxygenase (15-LOX-1) converts LTs into lipoxins (LXs), pro-resolving lipid mediators. LXs are involved in the attenuation of inflammation and cancer development. Purpose: We aimed to investigate the lipid mediator pathways, especially the LTs and LXs pathways, by studying 5-LOX and 15-LOX-1 expression in human cholangiocarcinoma (CCA) tissue. We also investigated the efficiency of zileuton (5-LOX inhibitor) treatment and BML111 (LXA4 analog) addition on CCA cell lines properties.

Patients and methods: The expression of 5-LOX and 15-LOX-1 in fifty human cholangiocarcinoma (CCA) tissue was analyzed using immunohistochemical staining. In addition, the effect of zileuton and BML-111 on CCA cell growth and migration was demonstrated using a cell viability assay and wound-healing assay, respectively. Furthermore, the molecular mechanism by which zileuton inhibits CCA cell migration was revealed using immunofluorescent staining and western blot analysis, respectively.

Results: We demonstrate that the upregulation of 5-LOX is significantly correlated with CCA recurrent status. A positive 15-LOX-1 signal was significantly associated with a longer survival time in CCA patients. We found that co-expression of 5-LOX and 15-LOX-1 resulted in a relatively good prognosis in CCA patients. In addition, zileuton could inhibit CCA cell migration as well as BML-111. Interestingly, zileuton treatment not only downregulated 5-LOX, but also upregulated 15-LOX-1, together with reversing the epithelial-mesenchymal transition to mesenchymal-epithelial transition phenotype as observed in EMT marker western blot.

Conclusion: These findings suggest that 5-LOX and 15-LOX-1 play a key role in CCA and may serve as targets for CCA therapy.

Keywords: cholangiocarcinoma, 5-LOX, 15-LOX-1, zileuton, EMT, Akt pathway

\section{Introduction}

Cholangiocarcinoma (CCA) is a cancer arising from the bile duct epithelial cells. The highest incidence in the world is seen in northeastern Thailand, where liver fluke (Opisthorchis viverrini $[\mathrm{Ov}]$ ) infection is the major risk factor for its development. ${ }^{1}$ Chronic inflammation induced by $O v$ disturbs the homeostasis of oxidants/antioxidants and DNA repair enzymes by increasing levels of pro-inflammatory cytokines and nuclear factor $\mathrm{\kappa B}$ that control cyclooxygenase-2 $\left(\mathrm{COX}_{2}\right)$ and inducible nitric oxide activities. Oxidative and nitrative stress can induce the production of reactive oxygen and nitrogen species in inflamed target cells resulting in high levels of oxidized DNA and DNA bases modified by lipid peroxidation products. ${ }^{2}$ This suggests that $O v$ infection causing chronic BY
hereby accept the Terms. Non-commercial uses of the work are permitted without any further permission from Dove Medical Press Limited, provided the work is properly attributed. For permission hereby accept the Terms. Non-commercial uses of the work are permitted without any further permission from Dove Medice
for commercial use of this work, please see paragraphs 4.2 and 5 of our Terms (https://www.dovepress.com/terms.php). 
inflammation may lead to disorganization of the immune response contributing to cholangiocarcinogenesis. ${ }^{2}$

Lipid mediators, a class of bioactive lipids, are released via specific processes in response to tissue injury or infection, such as the entry of bacteria into the body. ${ }^{3}$ These mediators are divided into two groups, the proinflammatory lipid mediators, such as prostaglandins (PGs) and leukotrienes (LTs), and the pro-resolving lipid mediators, such as lipoxins (LXs) and resolvins (RVs). LTs and PGs amplify acute inflammation, whereas LXs have unique anti-inflammatory actions. ${ }^{4}$ Therefore, the regulation of lipid mediator class switching is very important for the control of inflammatory processes and for returning tissue to homeostasis. The failure of lipid mediator class switching leads to chronic inflammation and is often linked to pathogenesis in several diseases, including cancer. ${ }^{4}$ Currently, evidence is accumulating suggesting that a distinct set of proteins that are essential for signal transduction (receptors), transport (transporters), and biosynthesis (enzymes), such as cyclooxygenase-2 (COX-2, producing PGs), 5-lipoxygenase (5-LOX, generating LTs), and 15-lipoxygenase (15-LOX, converting LTs to be LXs), could be used to investigate the functions and dynamics of lipid mediators. ${ }^{3}$ However, 15 -LOX is composed of two isoforms, 15-LOX-1 and 15-LOX-2. A conversion of LTs to LX is done only by 15 -LOX-1, ${ }^{4}$ whereas 15 -LOX-2 converts arachidonic acid to $15 S$-hydroxyeicosatetraenoic acid. Hence, lipid mediator class switching is mainly regulated by the two enzymes 5-LOX and 15-LOX-1. ${ }^{5}$ Several studies have been performed to uncover the role(s) of lipid mediators in cancer development and progression. Tong et $\mathrm{al}^{6}$ demonstrated that LTB4 can induce pancreatic cancer cell proliferation. In contrast, Chen et $\mathrm{al}^{7}$ demonstrated that BML-111, which is a $\mathrm{LXA}_{4}$ analog, can inhibit tumor growth in the mouse model. There are numerous studies relating high 5-LOX expression to a poor prognosis in cancers, including colorectal, pancreatic, and colon cancers. ${ }^{8-10}$ Moreover, 15-LOX isoforms, including 15-LOX-1 and 15-LOX-2, are correlated with tumor suppression in many cancers. ${ }^{11}$ In particular, for 15-LOX-1, it has been found that low expression is correlated with a poor prognosis in various cancers, including esophageal, pancreatic, colon, and colorectal cancers. ${ }^{12-15}$ We recently showed that a high expression of $\mathrm{COX}_{2}$ could be observed in CCA tissues and that $\mathrm{PGE}_{2}$ promotes the proliferation and migration of CCA cell lines, ${ }^{16}$ although the importance of the LOX pathways in CCA is still unknown.

Based on these data, LOX might be a crucial enzyme playing a vital function in CCA development. Therefore, we investigated the expression of 5-LOX (the key enzyme generating LTs) and 15-LOX-1 (the key enzyme generating
LXs) in the progression of CCA to determine whether these can be used as therapeutic targets for CCA treatment.

\section{Materials and methods Human CCA tissue specimens}

Fifty samples of CCA tissue were collected from CCA patients admitted to the surgical wards of Srinagarind Hospital, Khon Kaen University. These were kept by the Cholangiocarcinoma Research Institute. The protocol for the collection was approved by the Ethics Committee for Human Research, Khon Kaen University (\#HE571283). Written informed consent was obtained from all patients.

The clinicopathological features of all samples are shown in Table 1. There were 20 samples from female cases (40\%) and 30 samples from male cases $(60 \%)$. The median age of the patients was 56 years (range 36-76 years). The histological types were 38 cases of nonpapillary (76\%) and 12 cases of papillary CCA. Based on the TNM staging, there were 12 cases of stage I-II (24\%) and 28 cases of stage III-IV (76\%). There were 40 cases without metastases (80\%) and 10 cases with metastases. Among them, the recurrence status was clearly recorded in 41 cases, comprising 31 cases of recurrence $(76 \%)$ and 10 cases of nonrecurrence $(24 \%)$.

\section{Human CCA cell lines}

An immortalized cholangiocyte cell line MMNK1 and KKU-023, KKU-213 and KKU-100 CCA cell lines were used for this study. MMNK1 was received from Professor Naoya Kobayashi (Okayama University, Okayama, Japan). This cell line was established from cholangiocyte hepatocytes (transduced with SV40T and hTERT). ${ }^{17}$ KKU-023 cells were established from pathologically proven bile duct cancer tissues of a patient who was resident in the northeast Thailand and from whom written informed consent was obtained. ${ }^{18}$ The KKU-213 and KKU-100 cell lines were obtained from the Japanese Collection of Research Bioresources (JCRB) Cell Bank, Osaka, Japan. All of the cell lines were cultured in DMEM (Sigma-Aldrich Co., St Louis, MO,

Table I The patient's clinicopathological data

\begin{tabular}{l|l}
\hline Patients (n=50) & Mean or percentage \\
\hline Age (years) & $56 \pm 10(36-76)$ \\
Male (n) & $30 / 50(60 \%)$ \\
Histology (n) & \\
Nonpapillary type & $38(76 \%)$ \\
Papillary type & $12(24 \%)$ \\
Stage (I-II) & $12(24 \%)$ \\
Stage (III-IV) & $38(76 \%)$ \\
Survival (days) & $378 \pm 500(34-2,580)$ \\
Patients with metastases (n) & $10 / 50(20 \%)$ \\
\hline
\end{tabular}


USA) supplemented with inactivated $10 \% \mathrm{FBS}, 100 \mathrm{U} / \mathrm{mL}$ of penicillin-streptomycin, and $\mathrm{NaHCO}_{3}$ in a humidified atmosphere containing $5 \% \mathrm{CO}_{2}$.

\section{Chemicals}

Zileuton was purchased from Sigma-Aldrich Co., and BML111 was purchased from Abcam (Cambridge, UK).

\section{Immunohistochemical staining}

Immunohistochemical staining was performed to identify the expression of 5-LOX and 15-LOX-1 in human CCA tissues. Paraffin-embedded tissues were de-paraffinized and rehydrated with xylene and ethanol, respectively. Antigen retrieval was performed by pressure cooking with Tris-EDTA pH 9.0 containing $0.05 \%$ Tween 20 for 3 minutes. The sections were treated with $0.3 \%$ hydrogen peroxide to block endogenous hydrogen peroxide activity and $10 \%$ skim milk to block nonspecific binding for 30 minutes each. The primary antibody direct anti-5-LOX (diluted 1:50; ab169755; Abcam) and anti15-LOX-1 (diluted 1:150; ab119774; Abcam) were incubated for 1 hour at room temperature followed by $4^{\circ} \mathrm{C}$ overnight. The sections were then incubated with peroxidase-conjugated Envision TM secondary anti-rabbit antibody (Dako Denmark A/S, Glostrup, Denmark) for 1.30 hour for 5-LOX antibody and with secondary antibody for 3 hours for 15-LOX-1 antibody after washing with $0.1 \%$ (v/v) Tween 20 in PBS (3 times) and PBS for 5 minutes. After that, the signal was developed with a 3,3'diaminobenzidine tetrahydrochloride (DAB) substrate kit (Vector Laboratories, Inc., Burlingame, CA, USA) for 15 minutes and the slides were then counterstained with Mayer's hematoxylin. Finally, the sections were dehydrated with ethanol and xylene and mounted with permount (Bio Optica Milano S.p.A., Milano, Italy). Next, the stained sections were viewed under a microscope using $\times 400$ magnification $(\mathrm{Ni}-\mathrm{U}$; Nikon Instruments, Melville, NY, USA). The scoring of immunohistochemistry (IHC) was determined using $H$-score. ${ }^{19}$

\section{Immunofluorescent staining}

Immunofluorescent staining was performed to detect the protein localization of 5-LOX and 15-LOX-1 in CCA tissues. E-cadherin and vimentin (an epithelial-mesenchymal transition [EMT] marker) were also observed by immunofluorescent staining in the zileuton-treated cell line (KKU-023). Protein localization of 5-LOX and 15-LOX-1 in CCA tissues was performed by immunohistochemical staining until primary antibody addition. The tissue was then incubated with Alexa Fluor 488 or 555-conjugated secondary antibodies (Thermo Fisher Scientific, Waltham, MA, USA) (dilution 1:100 for 5-LOX and 1:300 for 15-LOX-1) for 1 hour in the dark.
Mounting was then carried out in mounting medium (1:200 DAPI in 50\% glycerol PBS), and staining was detected under a confocal microscope (Carl Zeiss Meditec AG, Jena, Germany). For EMT expression, KKU-023 cells were treated with 50,100 , and $200 \mu \mathrm{M}$ of zileuton. The cells were washed with PBS and then fixed by the addition of $4 \%$ paraformaldehyde for 30 minutes followed by washing twice with PBS at room temperature. The blocking step was performed using 5\% skim milk for 1 hour at room temperature, and the cells were then incubated with primary E-cadherin and vimentin (dilution 1:100 for all). After incubation, they were washed three times with PBS and incubated with 1:200 secondary antibody (fluoresce dye) for 1 hour in the dark. Finally, the cells were washed three times with PBS followed by mounting in medium (1:200 DAPI in 50\% glycerol PBS) and staining was detected under a confocal microscope using $\times 630$ magnification.

\section{SDS-PAGE and Western blot analysis}

Protein extracts were loaded onto NuPAGE ${ }^{\circledR}$ Novex 4\%-12\% Bis-Tris gels (Thermo Fisher Scientific) using MES running buffer (Thermo Fisher Scientific) and blotted onto PVDF membranes (Bio-Rad Laboratories Inc., Hercules, CA, USA). The membranes were blocked with 5\% skim milk in Trisbuffered saline (TBS) at room temperature for 1 hour. Then, they were stained with primary antibody at $4{ }^{\circ} \mathrm{C}$ overnight. After that, they were washed with TBS containing $0.1 \%$ Tween 20 (three times) followed by TBS for 5 minutes each. The membranes were further incubated with secondary antibody at room temperature for 1 hour and then washed. The signal was seen after membrane exposure to the ECL Prime Western Blotting Detection System (GE Healthcare, UK) by chemiluminescent detection, Amersham Imager 600 (GE Healthcare UK Ltd, Little Chalfont, UK). In this study, anti$\beta$-actin antibody was used as an internal loading control.

\section{Cell viability assay}

A sulforhodamine B (SRB) assay was used to determine cell proliferation. CCA cell lines were seeded in triplicate into 96-well plates and incubated or 72 hours using the following three media: control medium, medium containing varying concentrations of zileuton, and medium containing varying concentrations of BML-111. The treated cells were fixed with TCA and stained with $0.4 \%(\mathrm{w} / \mathrm{v}) \mathrm{SRB}$ in $1 \%$ (v/v) acetic acid for 45 minutes. They were subsequently washed three times with $1 \%(\mathrm{v} / \mathrm{v})$ acetic acid and dried. Next, the protein-bound stain was solubilized with $10 \mathrm{mM}$ Tris base $(\mathrm{pH} 10.5)$ for 1 hour using a shaker. Absorbance was measured at $540 \mathrm{~nm}$ using a microplate reader (Sunrise, TECAN Trading, Switzerland). 


\section{Wound-healing assay}

After the monolayer of CCA cells reach confluence, they were scratched by using a sterile plastic micropipette tip and then the cell debris removed. Cells were treated with medium with/without zileuton or BML-111. KKU-023 cells were observed at 12,18 , and 24 hours. KKU-213 cells were observed at 6,9 , and 12 hours, and KKU-100 cells were observed at 24,48 , and 72 hours. The migration of cells into the wound area were observed and photographed under a microscope using $\times 100$ magnification $(\mathrm{Ni}-\mathrm{U})$. The degree of cell spread as determined by wound area was compared between groups.

\section{Statistical analysis}

Statistical analysis was performed using the SPSS software (SPSS software v.19; IBM Corporation, Armonk, NY, USA).
The correlation between 5-LOX and 15-LOX-1 expression and the clinicopathological data of CCA patients were analyzed by Chi-squared test. Survival analysis was determined using Kaplan-Meier statistics. The graph was expressed as mean \pm SD (GraphPad Prism 5). The result of functional and nonfunctional assays, such as cell proliferation and migration, was determined using a Student's $t$-test (Microsoft excel 2013). The results were considered to be significant at $P<0.05$.

\section{Results}

\section{5-LOX and I5-LOX expression and their clinical associations}

The immunohistochemical staining of 5-LOX on 50 human CCA tissues showed that 5-LOX was expressed on perinuclease and the cytoplasm of cancer epithelial bile duct cells (Figure 1C), as well as on inflammatory cells (Figure 1A).

\section{A}

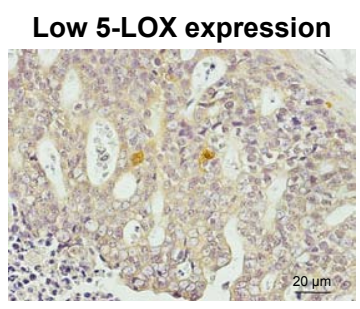

C

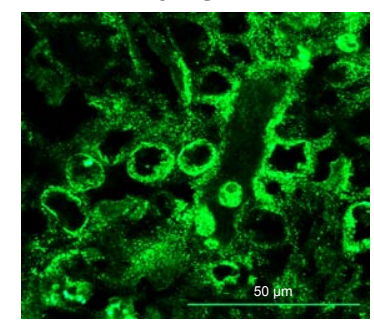

D

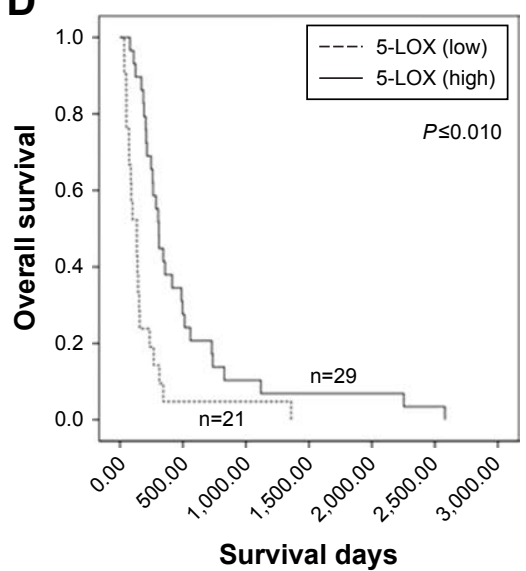

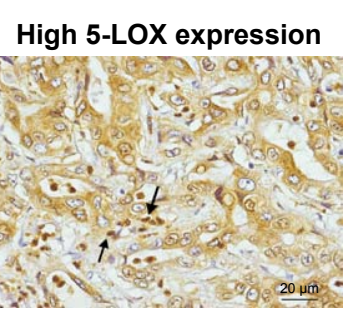

15-LOX-1

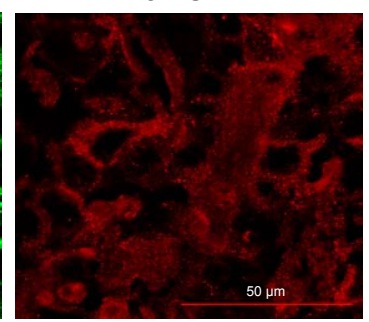

E

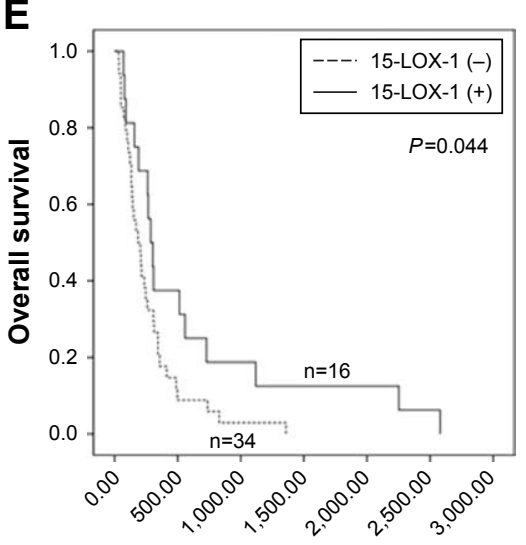

Survival days

DAPI
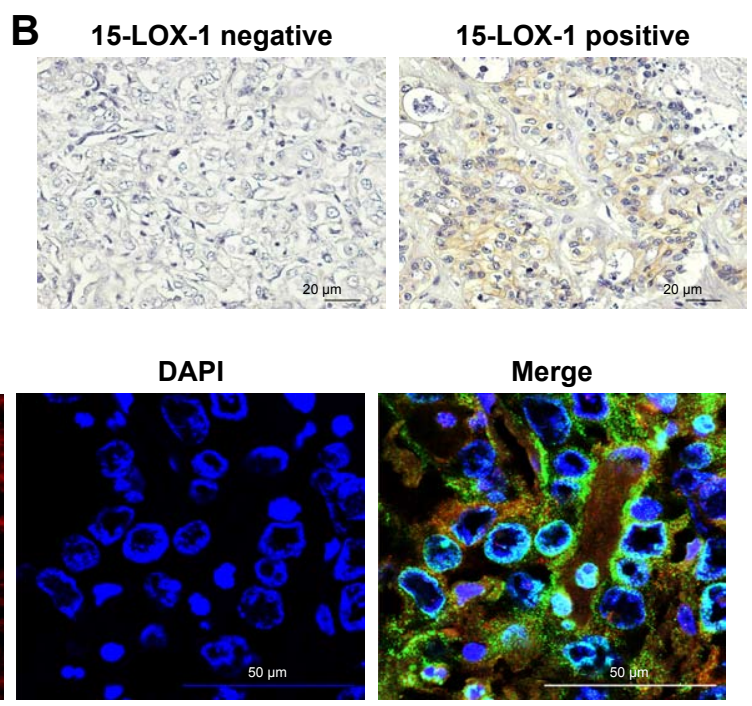

$\mathbf{F}$

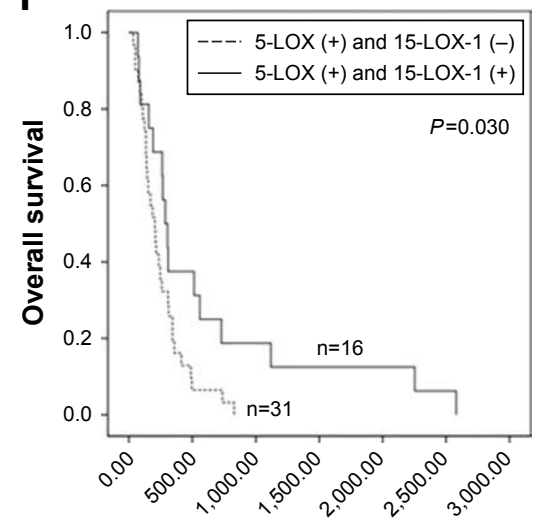

Survival days

Figure I The expression and localization of 5-LOX and I5-LOX-I in CCA tissues and their correlation with prognosis.

Notes: (A) Representative images of IHC demonstrating the high and low expression of 5-LOX in CCA tissues. Positive inflammatory cell staining was observed as indicated by the black arrow (magnification, $\times 400$ ). (B) The negative and positive signals of I5-LOX-I in CCA tissues were determined by IHC staining (magnification, $\times 400)$. (C) The co-localization of 5-LOX and I5-LOX-I immunofluorescence staining in CCA tissues was evaluated (magnification, $\times 400$ ). (D-F) Survival analysis of 5-LOX, I5-LOX-I, and combination of 5-LOX and I5-LOX-I expression in each patient with CCA by the Kaplan-Meier method.

Abbreviations: CCA, cholangiocarcinoma; IHC, immunohistochemistry; 5-LOX, 5-lipoxygenase; I5-LOX-I, I5-lipoxygenase. 
Table 2 Univariate analysis for 5-LOX and I5-LOX-I expression in human CCA tissues and the patient's clinicopathological data

\begin{tabular}{|c|c|c|c|c|c|c|c|}
\hline \multirow[t]{2}{*}{ Characteristics } & \multirow[t]{2}{*}{$\mathbf{n}$} & \multicolumn{2}{|c|}{ 5-LOX } & \multirow[t]{2}{*}{$P$-value } & \multicolumn{2}{|l|}{ I5-LOX } & \multirow[t]{2}{*}{$P$-value } \\
\hline & & Low & High & & Negative & Positive & \\
\hline Age (years) & & & & 0.077 & & & 0.846 \\
\hline$<56$ & 26 & 14 & 12 & & 18 & 8 & \\
\hline$\geq 56$ & 24 & 7 & 17 & & 16 & 8 & \\
\hline Sex & & & & $0.047^{\mathrm{a}}$ & & & 0.804 \\
\hline Female & 20 & 5 & 15 & & 14 & 6 & \\
\hline Male & 30 & 16 & 14 & & 20 & 10 & \\
\hline Histological types & & & & 0.520 & & & 0.728 \\
\hline Nonpapillary & 38 & 15 & 23 & & 25 & 13 & \\
\hline Papillary & 12 & 6 & 6 & & 9 & 3 & \\
\hline Metastasis & & & & 1.000 & & & 1.000 \\
\hline Nonmetastasis & 40 & 17 & 23 & & 27 & 13 & \\
\hline Metastasis & 10 & 4 & 6 & & 7 & 3 & \\
\hline TNM stage & & & & 0.193 & & & 0.700 \\
\hline I-II & 8 & 2 & 6 & & 5 & 3 & \\
\hline III-IV & 42 & 21 & 21 & & 29 & 13 & \\
\hline Recurrence & & & & $0.032^{\mathrm{a}}$ & & & 0.700 \\
\hline Nonrecurrence & 10 & 8 & 2 & & 8 & 22 & \\
\hline Recurrence & 31 & 2 & 19 & & 2 & 9 & \\
\hline Tumor size $(\mathrm{cm})$ & & & & 0.183 & & & 0.409 \\
\hline$<2$ & 2 & 0 & 2 & & 2 & 0 & \\
\hline$\geq 2$ & 31 & 15 & 16 & & 23 & 8 & \\
\hline
\end{tabular}

Note: ${ }^{a} P<0.05$ indicates a statistically significant difference.

Abbreviations: CCA, cholangiocarcinoma; 5-LOX, 5-lipoxygenase; 15-LOX-I, I5-lipoxygenase.

Twenty-one of the 50 CCA patients (42\%) showed a low expression of 5-LOX, while high expression was seen in 29 cases $(58 \%)$ (Figure 1A). Table 2 shows that significantly more male than female $(P=0.047)$ CCA patients have a high 5-LOX signal. Importantly, the CCA patients with a high level of 5-LOX were significantly associated with the recurrence of disease $(P=0.032)$ (Table 2). Kaplan-Meier survival analysis showed that patients with 5-LOX overexpression have a significantly longer survival rate than low expression patients $(P<0.010$; Figure 1D).

A positive signal of $15-\mathrm{LOX}-1$ was seen in 16 cases $(32 \%)$ (Figure 1C), whereas negative expression was observed in 34 cases (68\%) (Figure 1B). Positive 15-LOX-1 expression was not significantly associated with any other clinical parameters (Table 2). Interestingly, patients with a high expression of 15-LOX-1 had a significantly longer survival when compared with the patients with a low 15-LOX-1 expression ( $P=0.044$; Figure 1E). Combining 5-LOX and 15-LOX-1 expression revealed a significant correlation between the 5-LOX-positive and 15-LOX-1-negative groups with shorter survival rate than the 5-LOX-positive and 15-LOX-1-positive groups ( $P=0.030$; Figure $1 \mathrm{~F})$.

\section{Effect of zileuton and BML- I I I on CCA cell viability}

The level of 5-LOX and 15-LOX-1 in cell lines determined by Western blot revealed the expression of 5-LOX in
KKU-023, while it was not found in MMNK-1, KKU-213, or KKU-100. A high signal for 15-LOX-1 was observed in KKU-023 and KKU-213, whereas a low signal was found in MMNK-1 and no signal was found in KKU-100 (Figure 2A). Importantly, the SRB assay showed that CCA cell viability was inhibited by zileuton at 72 hours at $400 \mu \mathrm{M}$ in all CCA cell lines (Figure 2B). However, low concentrations of zileuton did not affect CCA cell survival. Similarly, BML-111 did not affect CCA cell viability in any of the CCA cell lines (Figure 2C).

\section{Zileuton inhibits CCA cell migration}

The ability of cancer cells to migrate in KKU-023 was significantly decreased after zileuton treatment in a dosedependent manner at 18 hours $(P<0.05)$ and 24 hours $(P<0.05)$, while cell migration at 12 hours was inhibited at 100 and $200 \mu \mathrm{M}$ of zileuton $(P<0.01)$ (Figure $3 \mathrm{~A}$ and $\mathrm{B})$. In addition, there was a significant inhibition of KKU-213 cell migration in a dose-dependent manner after treatment with zileuton at 12 hours, while decreasing cell migration at $200 \mu \mathrm{M}$ of zileuton was found at 9 hours $(P<0.05)$ (Figure $3 \mathrm{C}$ and D). Furthermore, cancer cell migration in KKU-100 treated with 50, 100, and $200 \mu \mathrm{M}$ of zileuton was significantly suppressed at 48 hours $(P<0.05)$, as was cell migration at 100 and $200 \mu \mathrm{M}$ of zileuton at 24 and 72 hours (Figure $3 \mathrm{E}$ and $\mathrm{F}$ ). 


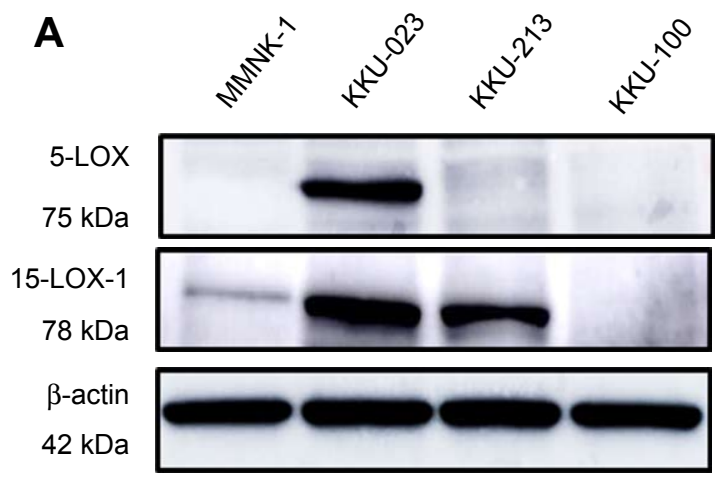

B

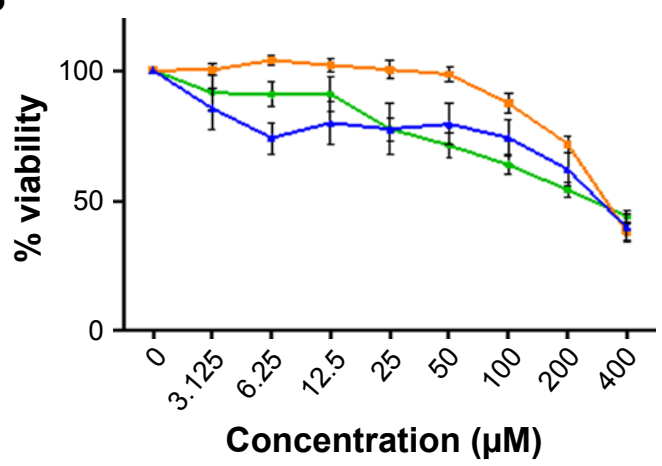

C

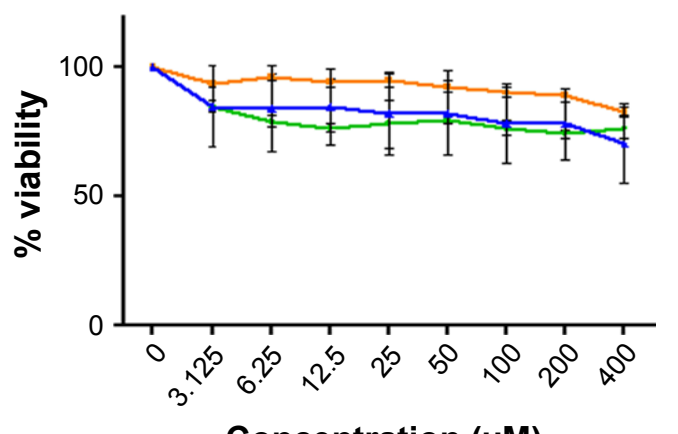

Concentration $(\mu \mathrm{M})$

$$
\rightarrow \mathrm{KKU}-023-\mathrm{KKU}-213 \mp \mathrm{KKU}-100
$$

Figure 2 The expression of 5-LOX and 15-LOX-I in CCA cell lines and the proliferation of CCA cell lines after treatment with zileuton or BML-I II.

Notes: (A) Western blot analysis of 5-LOX and I5-LOX-I expression in KKU-023, KKU-I00, and KKU-2I3 CCA cell lines, compared to immortalized cholangiocytes, MMNKI. (B) The percentage of CCA cell survival after treatment with different concentrations of zileuton for 72 hours on three CCA cell lines. (C) The percentage of CCA cell survival after treatment with different concentrations of BML-III for 72 hours on three CCA cell lines. All data are expressed as mean \pm SD of two independent experiments.

Abbreviations: CCA, cholangiocarcinoma; 5-LOX, 5-lipoxygenase; I5-LOX-I, I5lipoxygenase.

\section{Migration of CCA cell line treated with BML-I I I}

Cell migration of KKU-023 could be inhibited by BML-111 at a concentration of $50 \mu \mathrm{M}(P<0.05)$ (Figure $4 \mathrm{~A}$ and $\mathrm{B}$ ). KKU-100 cell migration was significantly decreased after treatment with 50 and $100 \mu \mathrm{M}$ of BML-111 at 72 hours
$(P<0.05)$; additionally, $200 \mu \mathrm{M}$ of BML-111 could inhibit migration at 24 and 48 hours $(P<0.05)$, and 72 hours $(P<0.01)$ (Figure 4E and F). However, no inhibitory effect of BML-111 on KKU-213 cell migration was found (Figure 4C and D).

\section{Zileuton reduces the expression of EMT-related protein possibly via the Akt signaling pathway}

Western blot analysis was performed to determine protein expression of E-cadherin, vimentin, snail, and also targeted protein consisting of 5-LOX and 15-LOX-1 in KKU-023. The expression of 5-LOX was decreased after treatment with 100 and $200 \mu \mathrm{M}$ of zileuton, while the 15-LOX-1 level was increased after treatment with 50,100 , and $200 \mu \mathrm{M}$ of zileuton (Figure 5A and B, respectively). Moreover, E-cadherin was upregulated, whereas vimentin and snail were decreased in a dose-dependent manner. In addition, zileuton-treated KKU023 cells showed enhanced signal of E-cadherin, while the vimentin signal of zileuton-treated cells was lower than that of untreated cells (Figure 5C). Interestingly, the Akt signaling pathway was inhibited in zileuton-treated KKU-023 cells. The suppression of the Akt signaling pathway was also observed in zileuton-treated KKU-100 cells (data not shown).

\section{Discussion}

LTs are the lipid mediators that are involved in inflammatory processes and carcinogenesis. ${ }^{20-23}$ LTs have been shown to play an important role in many types of cancer. ${ }^{24-27} 5$-LOX is a key enzyme generating LTs. ${ }^{28}$ Upregulation of 5-LOX correlates with a poor prognosis and cancer progression in several cancers. ${ }^{8-10}$ The inhibition of 5-LOX results in a reduction of cancer progression, including cell proliferation, migration, invasion, and angiogenesis. ${ }^{29} 15-\mathrm{LOX}-1$ is an enzyme that converts LTs into LXs. ${ }^{30}$ LXs are the lipid mediators that are involved in the resolution of inflammation and cancer. ${ }^{30}$ They have been found to suppress tumor growth migration and angiogenesis. ${ }^{7,31,32}$ Downregulation of $15-\mathrm{LOX}-1$ is correlated with a poor prognosis in many cancers. ${ }^{12-15}$ However, the roles of 5-LOX and 15-LOX-1 in CCA cell proliferation and metastasis are still unknown. Therefore, understanding the function of 5-LOX and 15-LOX-1 in CCA might lead to targets for a new CCA treatment.

There was a high expression of 5-LOX in human CCA tissues that was correlated with disease recurrence. These data were consistent with a previous study showing that 5-LOX is critical for the maintenance of cancer stem cell (CSC)-like cells in a model of chronic myeloid leukemia. ${ }^{33}$ Inhibition of 
A

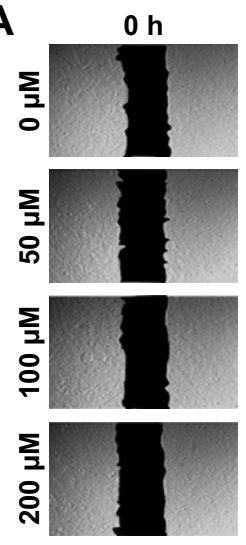

C

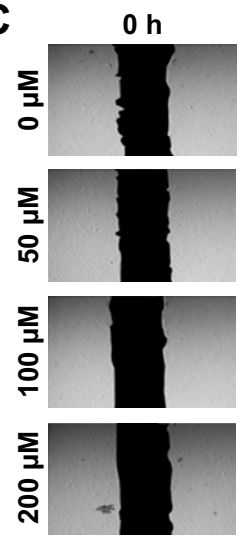

E
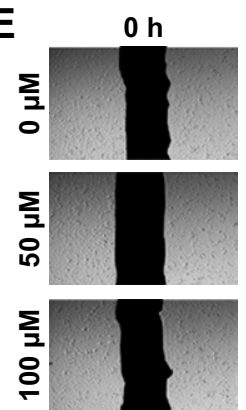

్ㅣㅇ

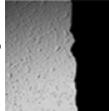

$12 \mathrm{~h}$
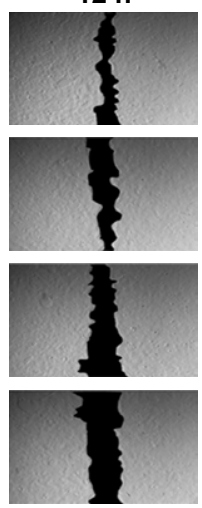

$6 \mathrm{~h}$
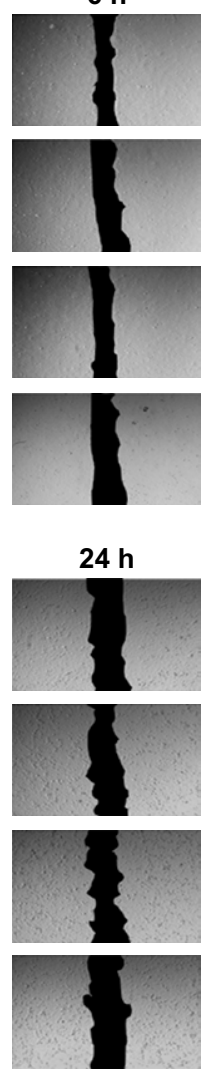

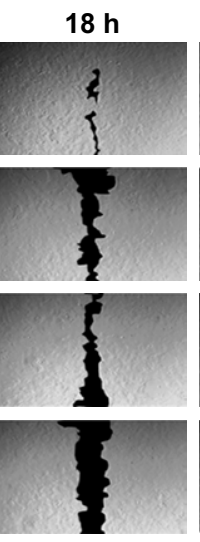

$9 \mathrm{~h}$
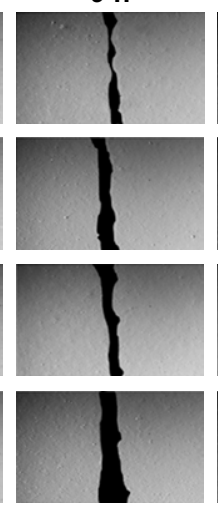

72 h
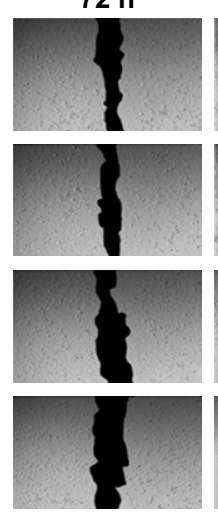

$24 \mathrm{~h}$

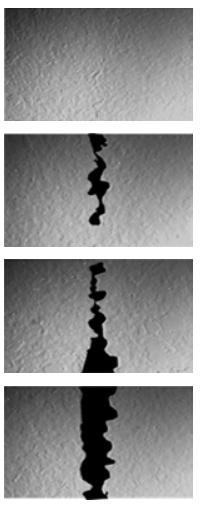

$12 \mathrm{~h}$
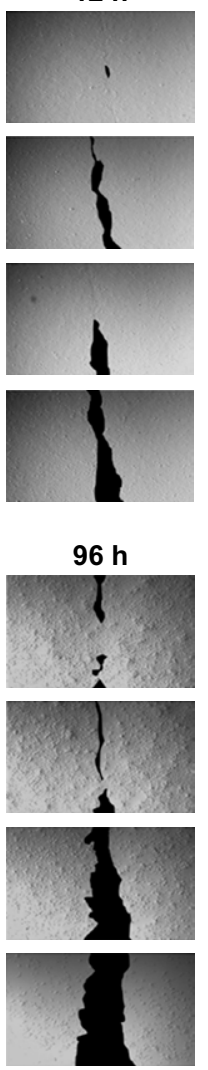

B

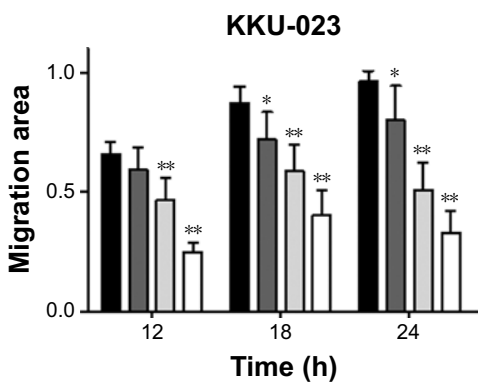

D

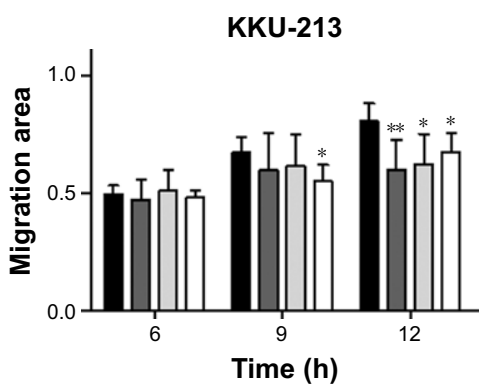

$\mathbf{F}$

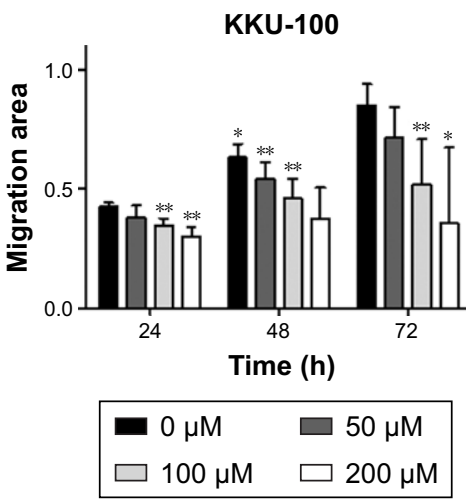

Figure 3 The migration of CCA cell lines with the presence/absence of zileuton.

Notes: (A, C, and E) Micrographs of wound healing assays for 50, 100, and $200 \mu$ M of zileuton-treated KKU- $023, \mathrm{KKU}-213$, and KKU-I00 cells, respectively (magnification, $\times 100$ ). (B, D, and F) Percentage of wound closure for the corresponding cell lines including KKU-023, KKU-2I3, and KKU-I00 cells, respectively. Data were obtained from two independent experiments $(* P<0.05$ and $* * P<0.01)$.

Abbreviation: CCA, cholangiocarcinoma.

5-LOX downregulated the stemness property in prostate cancer stem cells. ${ }^{34}$ We revealed that a high 5-LOX expression was correlated with a good prognosis in CCA patients. We further investigated 15-LOX-1 expression in human CCA tissues. Most CCA patients have a low expression of 15-LOX-1, and patients with a negative-signal were associated with a poor prognosis. Similar to other studies, downregulation of 15-LOX-1 correlated with decreased survival in several types of cancer ${ }^{12,13,15}$ and restoration of 15-LOX-1 expression promoted pro-resolving lipid mediator production that could trigger apoptosis in colorectal cancer. ${ }^{13,14} \mathrm{We}$ hypothesize that in cases positive for both 5-LOX and 15-LOX-1, patients should have a good prognosis. Therefore, we investigated 5-LOX and 15-LOX-1 in each CCA sample. Our findings 
A
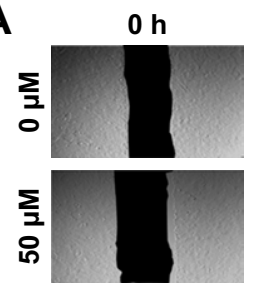

릉

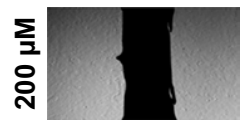

C

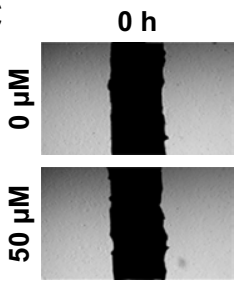

$\stackrel{\Sigma_{2}}{\stackrel{2}{2}}$

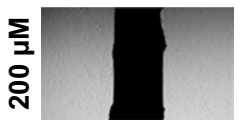

E
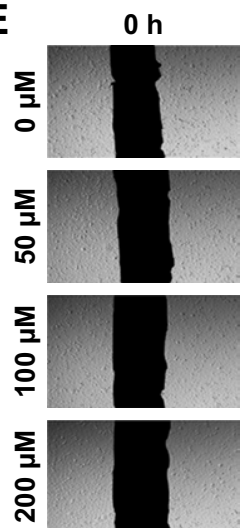

$12 \mathrm{~h}$
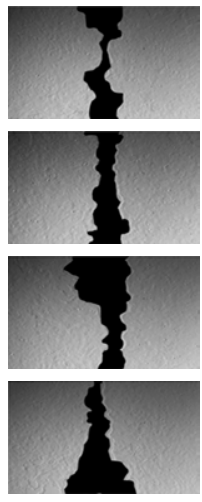

$6 \mathrm{~h}$
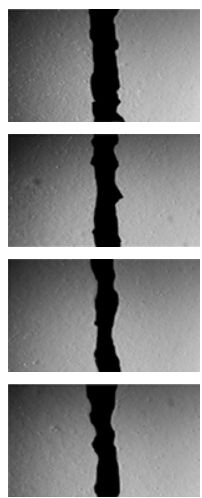

$24 \mathrm{~h}$
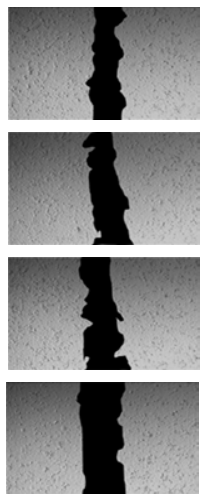
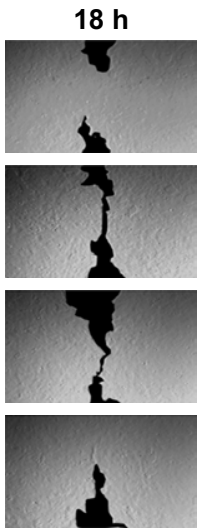

$9 \mathrm{~h}$
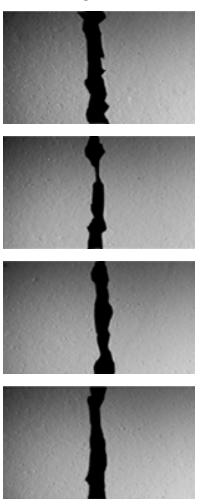

$72 \mathrm{~h}$
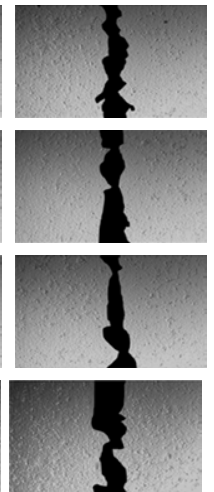

$24 \mathrm{~h}$
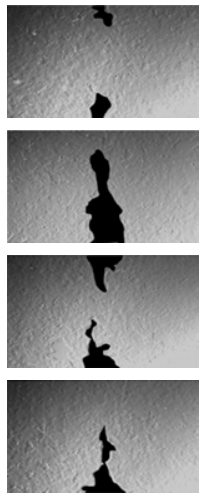

$12 \mathrm{~h}$
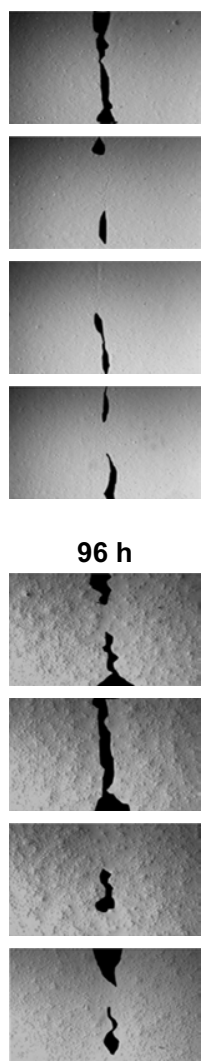

B

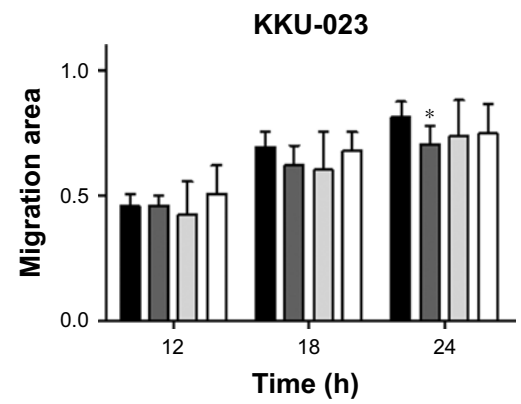

D
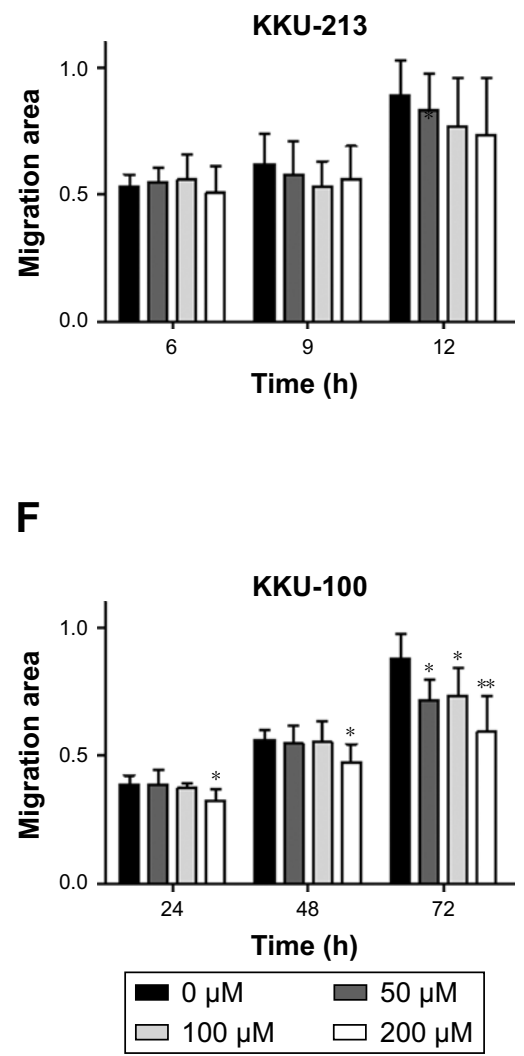

Figure 4 Migration of CCA cell line treated with BML-I I I.

Notes: The migration of CCA cell lines with a presence/absence of BML-I I I. (A, C, and E) Micrographs of wound healing assays for 50, I00, and 200 $\mu$ M of BML-I I -treated KKU-023, KKU-2I3, and KKU-I00 cells, respectively (magnification, $\times 100$ ). (B, D, and F) Percentage of wound closure for the corresponding cell lines including KKU-023, KKU-2I3, and KKU- 100 cells, respectively. Data were obtained from two independent experiments $(* P<0.05$ and $* * P<0.01)$.

Abbreviation: CCA, cholangiocarcinoma.

indicate that co-expression of 5-LOX and 15-LOX-1 did correlate with a good prognosis, while the CCA patients with 5-LOX expression and a negative signal for 15-LOX-1 correlated with a poor prognosis. Thus, 5-LOX and 15-LOX-1 might be important targets for CCA treatment. We next examined the effect of zileuton and BML-111 on CCA cell proliferation and migration in an in vitro model.
Our study suggests an inhibitory effect of zileuton on CCA cell proliferation at high concentrations. Previous studies demonstrated that zileuton can suppress cell proliferation and induce apoptosis in many cancer types, such as pancreatic cancer, colon cancer, and hepatocellular carcinoma. ${ }^{35-37}$ However, BML-111 has no inhibitory effect on CCA cell proliferation. 
A

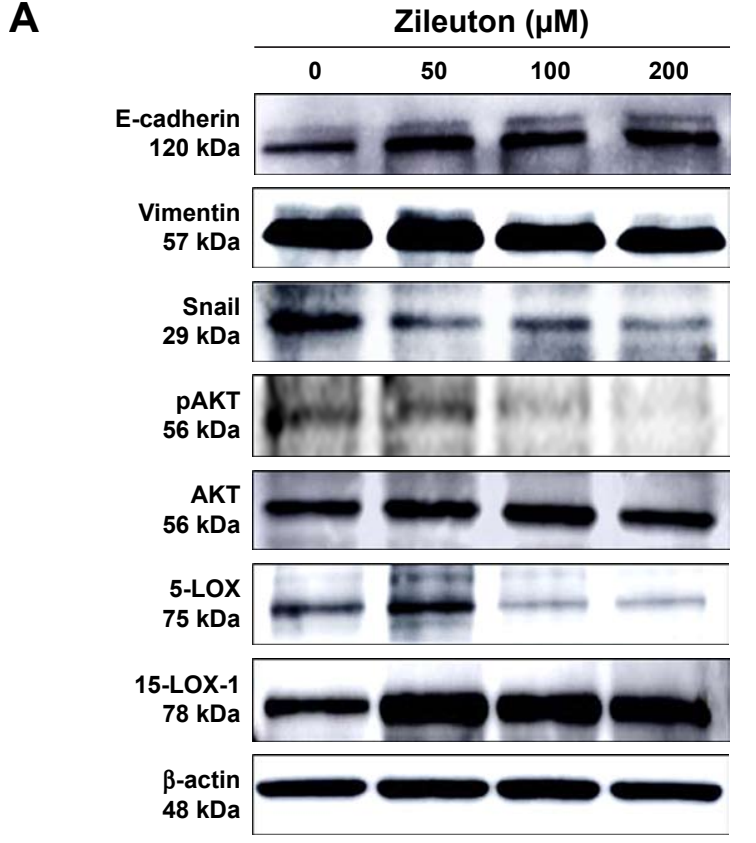

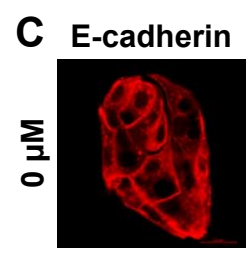
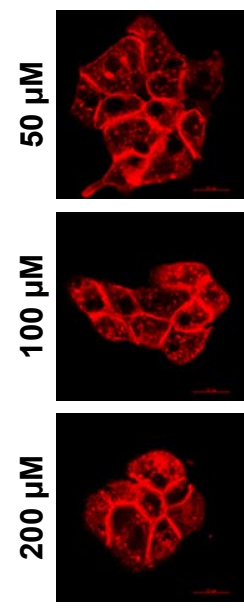
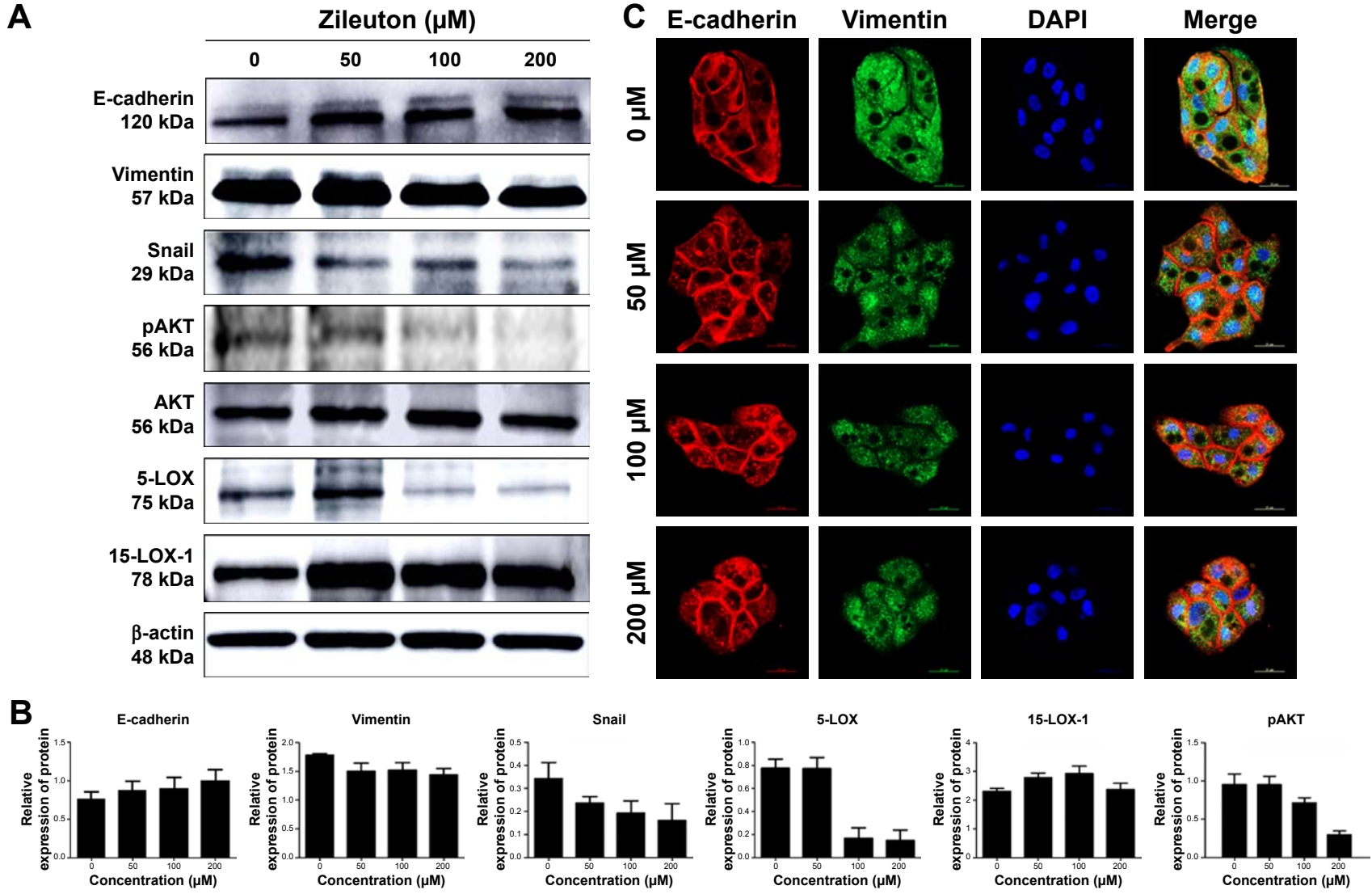

Figure 5 Western blot and immunofluorescence analyses for cell migration-related protein expression and 5-LOX and I5-LOX-I expression.

Notes: (A) Western blot analysis of E-cadherin, vimentin, snail, 5-LOX, and I5-LOX-I expression in KKU-023-treated cells. (B) The level of expression of E-cadherin, vimentin, snail, 5-LOX, I5-LOX-I, and PAKT. (C) Immunofluorescent of E-cadherin and vimentin expression in KKU-023-treated cells.

Abbreviations: 5-LOX, 5-lipoxygenase; I5-LOX-I, I5-lipoxygenase.

Interestingly, our findings indicate a suppressing effect of zileuton on CCA cells. Similarly, previous data showed that the inhibition of 5-LOX by zileuton could suppress cancer cell migration in cancer types, including prostate cancer. ${ }^{38}$ The inhibitory effect of zileuton on TGF- $\beta 1$-induced migration in astrocytes was also previously reported. ${ }^{39}$ Although inhibition of CCA cell migration after BML-11 treatment was not significantly affected, BML-111 did inhibit hepatocarcinoma cell metastasis in an in vivo study. ${ }^{40}$

This is the first study to clarify the molecular mechanism of zileuton treatment by Western blot and immunofluorescence techniques. One of the important metastatic processes is the $\mathrm{EMT}^{41,42}$ that plays a role during the early stages of migration and invasion by cancer cells. ${ }^{42}$ It is involved in cancer progression and metastasis in many cancers. ${ }^{43-45}$ The key markers used for EMT identification include E-cadherin, involving in the formation and maintenance of cell-cell adhesion (epithelial marker), ${ }^{46}$ vimentin, involving in the attachment, migration, and increased motility (mesenchymal marker), ${ }^{47}$ and snail protein, a transcription factor of EMTrelated proteins promoting the motility and metastatic spread of tumor cells. Our findings indicate that vimentin and snail proteins were decreased, while E-cadherin was increased after zileuton treatment.

The PI3K/Akt pathway plays an important role in promoting EMT and the motility of cancer cell lines in several cancers. ${ }^{48,49}$ In addition, it is widely known that Akt acts downstream of 5-LOX activity. ${ }^{50}$ This study shows that the zileuton-treated cell line showed that phosphorylation of Akt was reduced. There is a previous study indicating that zileuton can reduce the activity of PI3K.${ }^{51}$ As a result, zileuton could inhibit cell migration by interrupting the expression of vimentin and snail, as well as being inversely correlated with increasing E-cadherin, possibly via the Akt signaling pathway.

Taken together, our findings indicate a significant correlation between expression with a good prognosis linked to CCA suppression. 5-LOX and 15-LOX-1 should therefore be 
considered for CCA treatment. However, the role of 5-LOX in recurrence is still unclear, an issue that should be further investigated.

\section{Acknowledgments}

This research was supported by Invitation Research Grant (IN60258), a scholarship from the Faculty of Medicine, Khon Kaen University, Thailand, to SK, a grant of Khon Kaen University (KKU62), a grant from Cholangiocarcinoma Screening and Care Program (CASCAP-09), and a grant from the Thailand Research Fund (grant no RSA5980013) allocated to WL. We thank Professor Trevor N Petney for editing the article via the Publication Clinic KKU, Thailand.

\section{Author contributions}

All authors contributed toward data analysis, drafting and revising the paper and agree to be accountable for all aspects of the work.

\section{Disclosure}

The authors report no conflicts of interest in this work.

\section{References}

1. Sripa B, Pairojkul C. Cholangiocarcinoma: lessons from Thailand. Curr Opin Gastroenterol. 2008;24(3):349-356.

2. Yongvanit P, Pinlaor S, Bartsch H. Oxidative and nitrative DNA damage: key events in opisthorchiasis-induced carcinogenesis. Parasitol Int. 2012;61(1):130-135.

3. Murakami M. Lipid mediators in life science. Exp Anim. 2011;60(1): $7-20$.

4. Lee HN, Na HK, Surh YJ. Resolution of inflammation as a novel chemopreventive strategy. Semin Immunopathol. 2013;35(2):151-161.

5. Brash AR, Boeglin WE, Chang MS. Discovery of a second 15S-lipoxygenase in humans. Proc Natl Acad Sci U S A. 1997;94(12):6148-6152.

6. Tong WG, Ding XZ, Talamonti MS, Bell RH, Adrian TE. LTB4 stimulates growth of human pancreatic cancer cells via MAPK and PI-3 kinase pathways. Biochem Biophys Res Commun. 2005;335(3):949-956.

7. Chen $\mathrm{Y}, \mathrm{Hao} \mathrm{H}, \mathrm{He} \mathrm{S}$, et al. Lipoxin $\mathrm{A} 4$ and its analogue suppress the tumor growth of transplanted $\mathrm{H} 22$ in mice: the role of antiangiogenesis. Mol Cancer Ther. 2010;9(8):2164-2174.

8. Hennig R, Ding XZ, Tong WG, et al. 5-Lipoxygenase and leukotriene $B(4)$ receptor are expressed in human pancreatic cancers but not in pancreatic ducts in normal tissue. Am J Pathol. 2002;161(2):421-428.

9. Soumaoro LT, Iida S, Uetake H, et al. Expression of 5-lipoxygenase in human colorectal cancer. World J Gastroenterol. 2006;12(39): 6355-6360

10. Wasilewicz MP, Kołodziej B, Bojułko T, et al. Overexpression of 5-lipoxygenase in sporadic colonic adenomas and a possible new aspect of colon carcinogenesis. Int J Colorectal Dis. 2010;25(9):1079-1085.

11. Ivanov I, Kuhn H, Heydeck D. Structural and functional biology of arachidonic acid 15-lipoxygenase-1 (ALOX15). Gene. 2015;573(1): $1-32$.

12. Hennig R, Kehl T, Noor S, et al. 15-lipoxygenase-1 production is lost in pancreatic cancer and overexpression of the gene inhibits tumor cell growth. Neoplasia. 2007;9(11):917-926.

13. Heslin MJ, Hawkins A, Boedefeld W, et al. Tumor-associated downregulation of 15-lipoxygenase-1 is reversed by celecoxib in colorectal cancer. Ann Surg. 2005;241(6):941-947.
14. Shureiqi I, Wojno KJ, Poore JA, et al. Decreased 13-S-hydroxyoctadecadienoic acid levels and 15-lipoxygenase-1 expression in human colon cancers. Carcinogenesis. 1999;20(10):1985-1995.

15. Shureiqi I, Xu X, Chen D, et al. Nonsteroidal anti-inflammatory drugs induce apoptosis in esophageal cancer cells by restoring 15-lipoxygenase-1 expression. Cancer Res. 2001;61(12):4879-4884.

16. Jongthawin J, Chusorn P, Techasen A, et al. PGE2 signaling and its biosynthesis-related enzymes in cholangiocarcinoma progression. Tumour Biol. 2014;35(8):8051-8064.

17. Maruyama M, Kobayashi N, Westerman KA, et al. Establishment of a highly differentiated immortalized human cholangiocyte cell line with SV40T and hTERT. Transplantation. 2004;77(3):446-451.

18. Saensa-Ard S, Leuangwattanawanit S, Senggunprai L, et al. Establishment of cholangiocarcinoma cell lines from patients in the endemic area of liver fluke infection in Thailand. Tumour Biol. 2017;39(11): 1010428317725925.

19. Cohen DA, Dabbs DJ, Cooper KL, et al. Interobserver agreement among pathologists for semiquantitative hormone receptor scoring in breast carcinoma. Am J Clin Pathol. 2012;138(6):796-802.

20. Peters-Golden M, Henderson WR. Leukotrienes. N Engl J Med. 2007; 357(18):1841-1854.

21. Busse WW. Leukotrienes and Inflammation. Am J Respir Crit Care Med. 1998;157(6):S210-S213.

22. Massoumi R, Sjölander A. The role of leukotriene receptor signaling in inflammation and cancer. Scientific World J. 2007;7:1413-1421.

23. Kupczyk M, Kuna P. The role of leukotrienes in inflammation and leukotriene inhibitors. Pol Merkur Lekarski. 1999;7(39):85-93.

24. Hagmann W. Cell proliferation status, cytokine action and protein tyrosine phosphorylation modulate leukotriene biosynthesis in a basophil leukaemia and a mastocytoma cell line. Biochem J. 1994; 299(2):467-472.

25. Bortuzzo C, Hanif R, Kashfi K, Staiano-Coico L, Shiff SJ, Rigas B. The effect of leukotrienes B and selected HETEs on the proliferation of colon cancer cells. Biochim Biophys Acta. 1996;1300(3):240-246.

26. Mezhybovska M, Wikström K, Ohd JF, Sjölander A. The inflammatory mediator leukotriene D4 induces beta-catenin signaling and its association with antiapoptotic Bcl-2 in intestinal epithelial cells. J Biol Chem. 2006;281(10):6776-6784.

27. Venerito M, Kuester D, Harms C, Schubert D, Wex T, Malfertheiner P. Upregulation of leukotriene receptors in gastric cancer. Cancers. 2011;3(3):3156-3168.

28. Powell WS, Rokach J. Biochemistry, biology and chemistry of the 5-lipoxygenase product 5-oxo-ETE. Progress Lipid Res. 2005;44(2-3): 154-183.

29. Wang D, Dubois RN. Eicosanoids and cancer. Nat Rev Cancer. 2010; 10(3):181-193.

30. Serhan CN, Chiang N, van Dyke TE. Resolving inflammation: dual anti-inflammatory and pro-resolution lipid mediators. Nat Rev Immunol. 2008;8(5):349-361.

31. Jin Y, Arita M, Zhang Q, et al. Anti-angiogenesis effect of the novel anti-inflammatory and pro-resolving lipid mediators. Invest Ophthalmol Vis Sci. 2009;50(10):4743-4752.

32. Serhan CN, Krishnamoorthy S, Recchiuti A, Chiang N. Novel antiinflammatory - pro-resolving mediators and their receptors. Curr Top Med Chem. 2011;11(6):629-647.

33. Roos J, Oancea C, Heinssmann M, et al. 5-Lipoxygenase is a candidate target for therapeutic management of stem cell-like cells in acute myeloid leukemia. Cancer Res. 2014;74(18):5244-5255.

34. Sarveswaran S, Thamilselvan V, Brodie C, Ghosh J. Inhibition of 5-lipoxygenase triggers apoptosis in prostate cancer cells via downregulation of protein kinase C-epsilon. Biochim Biophys Acta. 2011; 1813(12):2108-2117.

35. Xu XM, Deng JJ, Yuan GJ, et al. 5-Lipoxygenase contributes to the progression of hepatocellular carcinoma. Mol Med Rep. 2011;4(6): $1195-1200$.

36. Zhou GX, Ding XL, Wu SB, et al. Inhibition of 5-lipoxygenase triggers apoptosis in pancreatic cancer cells. Oncol Rep. 2015;33(2):661-668. 
37. Melstrom LG, Bentrem DJ, Salabat MR, et al. Overexpression of 5-lipoxygenase in colon polyps and cancer and the effect of 5-LOX inhibitors in vitro and in a murine model. Clin Cancer Res. 2008;14(20): 6525-6530.

38. Meng Z, Cao R, Yang Z, Liu T, Wang Y, Wang X. Inhibitor of 5-lipoxygenase, zileuton, suppresses prostate cancer metastasis by upregulating E-cadherin and paxillin. Urology. 2013;82(6):1452.e7.

39. Huang XQ, Zhang XY, Wang XR, et al. Transforming growth factor $\beta 1$-induced astrocyte migration is mediated in part by activating 5-lipoxygenase and cysteinyl leukotriene receptor 1. J Neuroinflammation. 2012;9:145.

40. Xu F, Zhou X, Hao J, et al. Lipoxin A4 and its analog suppress hepatocarcinoma cell epithelial-mesenchymal transition, migration and metastasis via regulating integrin-linked kinase axis. Prostaglandins Other Lipid Mediat. 2018;137:9-19.

41. Zeisberg M, Neilson EG. Biomarkers for epithelial-mesenchymal transitions. J Clin Invest. 2009;119(6):1429-1437.

42. Boyer B, Vallés AM, Edme N. Induction and regulation of epithelialmesenchymal transitions. Biochem Pharmacol. 2000;60(8): 1091-1099.

43. Bates RC, Mercurio A. The epithelial-mesenchymal tansition (EMT) and colorectal cancer progression. Cancer Biol Ther. 2005;4(4):371-376.

44. Huang L, Wu RL, Xu AM. Epithelial-mesenchymal transition in gastric cancer. Am J Transl Res. 2015;7(11):2141-2158.
45. Gurzu S, Banias L, Bara T, Feher I, Bara T, Jung I. The EpithelialMesenchymal Transition Pathway in Two Cases with Gastric Metastasis Originating from Breast Carcinoma, One with a Metachronous Primary Gastric Cancer. Recent Pat Anticancer Drug Discov. 2018;13(1): $118-124$.

46. Pećina-Slaus N. Tumor suppressor gene E-cadherin and its role in normal and malignant cells. Cancer Cell Int. 2003;3(1):17.

47. Ivaska J, Pallari HM, Nevo J, Eriksson JE. Novel functions of vimentin in cell adhesion, migration, and signaling. Exp Cell Res. 2007; 313(10):2050-2062.

48. Grille SJ, Bellacosa A, Upson J, et al. The protein kinase Akt induces epithelial mesenchymal transition and promotes enhanced motility and invasiveness of squamous cell carcinoma lines. Cancer Res. 2003;63(9): 2172-2178.

49. Okui G, Tobiume K, Rizqiawan A, et al. AKT primes snail-induced EMT concomitantly with the collective migration of squamous cell carcinoma cells. J Cell Biochem. 2013;114(9):2039-2049.

50. Bishayee K, Khuda-Bukhsh AR. 5-lipoxygenase antagonist therapy: a new approach towards targeted cancer chemotherapy. Acta Biochim Biophys Sin. 2013;45(9):709-719.

51. Dahlin A, Qiu W, Litonjua AA, et al. The phosphatidylinositide 3-kinase (PI3K) signaling pathway is a determinant of zileuton response in adults with asthma. Pharmacogenomics J. Epub 2018 Jan 3.
OncoTargets and Therapy

\section{Publish your work in this journal}

OncoTargets and Therapy is an international, peer-reviewed, open access journal focusing on the pathological basis of all cancers, potential targets for therapy and treatment protocols employed to improve the management of cancer patients. The journal also focuses on the impact of management programs and new therapeutic agents and protocols on

\section{Dovepress}

patient perspectives such as quality of life, adherence and satisfaction. The manuscript management system is completely online and includes a very quick and fair peer-review system, which is all easy to use. Visit http://www.dovepress.com/testimonials.php to read real quotes from published authors. 